\title{
Health Disparity and Health Professional Education: A new approach
}

\author{
Authors \\ Professor Shaun Ewen \\ The University of Melbourne \\ Dr Jenny Barrett \\ The University of Melbourne \\ Dr Sarah Howell-Meurs \\ The University of Melbourne
}

\section{Correspondence}

Professor Shaun Ewen

Director, Melbourne Poche Centre for Indigenous Health

The University of Melbourne

Alan Gilbert Building; Level 2, 161 Barry Street Parkville, Victoria, Australia 3010 Email: shaun.ewen@unimelb.edu.au

Phone: +61383443389 


\section{Abstract}

In this paper a new theoretical framework for curricula is proposed as a means of advancing the potential for health professional graduates to contribute optimally in the contemporary world of complex health care. A new theoretical approach to curriculum is needed to provide for a comprehensive and integrated view of the diversity in people and populations. These subject areas require rigor and strength equivalent to the strong and well-established foundations of the biosciences and clinical practice. A rigorous and coherent approach would replace what is often an ad-hoc response to diverse and marginalized people and populations in existing curricula. The current approach has not had sufficient impact on health disparities.

This paper presents the case for adopting an 'Intersectional' framework for health professional curricula to move the voices and experiences of marginalized groups from the periphery to the center of the curriculum conversations. The pillars of that framework are described and illustrated with examples, and the implications and expected benefits of adopting such a framework are discussed. To illustrate the limitations of the current approach to curriculum development and the need for and likely benefits of the proposed framework, the paper draws on the situation of Australian Indigenous peoples.

\section{Key Words}

Health disparity Indigenous health Intersectionality Medical curriculum 


\section{Background}

Many efforts to include population diversity in health professional curricula have been compartmentalized, producing a course with a single focus. For example, there are courses that focus on aspects of culture [1-9] and others that focus on sexuality [10-11]. Such singular focuses may serve to obscure different experiences of health that each person brings to health professional interactions and may even promote stereotyping and simplistic generalizations [12].

There have also been various international responses to the increasingly diverse patient populations that require health professionals' attention [13-16]. The 'cultural competence' approach represents one attempt to broaden health professional curricula towards better inclusion of diversity $[2,3,17,18]$. 'Cultural safety', 'women's health' and even the contemporary 'slow' medical education movement [19] represent other attempts. Such approaches recognize the increased potential for discordance that accompanies diversity - discordance between the patient's health beliefs and experiences and the beliefs and experiences of the clinician. Discordance is understood to further compromise patient care and health outcomes, and either ignores or amplifies health disparities.

There is, however, a lack of evidence that these approaches adequately address students' long-term perceptions of diversity or doctor-patient relations, let alone that they reduce the cultural and ethnic disparities in health care $[12,14,15,20,21]$. It has been suggested that by, their nature, these approaches oversimplify diversity [12], and that they perpetuate stereotypes, diminishing the importance of other aspects of identity that impact on a person's health/experiences of health care.

There is a need for a more fundamental change at curriculum development level. We propose that the logic and strengths of the accepted biomedical science basis of health professional curricula are maintained within a new partnership framework. The new partner would be an equally logical and strong approach to 
diversity at the core of curricula. In medicine and other curricula, the biomedical science foundation was firmly established by Flexner's radical report in 1910 which reflected a determination to not only improve medical school education but to also educate the public, to enable people to "discriminate between the illtrained and the rightly trained physician" [22]. In their critical appraisal of the success of the Flexerian model, influential contemporary leaders in medical education have noted - given so many changes in the scientific, pharmacological and technological foundations of medicine - there is a need for a fundamentally different theoretical approach to the view of the patient and society that dominates current health professional curricula [23]. Our interest is in creating a framework for the education of health professionals who, being 'rightly trained' will no longer contribute to health disparities.

One international approach to addressing the persistent disparities in health status between Indigenous and other peoples in Australia, New Zealand and Canada has been the inclusion of Indigenous health content in the education and training of health care professionals. The focus on Indigenous populations recognizes the social contract that Medical Schools have with their local populations, and recognizes the need to address past injustices. It also acknowledges institutions as 'anchor' institutions, geographically embedded in a time and place. Well taught, the principles that underlie the teaching focus on Indigenous health should also be used within the curriculum and be applicable to other population groups. In Australia this response has occurred at three levels. First, the inclusion of this content has been mandated through professional accreditation requirements by bodies including the Australian Medical Council and the Australian Dental Council. Core competencies for professional programs have been endorsed by responsible bodies including the Council of Academic Public Health Institutions Australia (CAPHIA) for their Master of Public Health programs. Second, national networks including the Leaders in Indigenous Medical Education Network (LIME) and the Public Health Indigenous Leadership in Education (PHILE) have provided leadership to support and advocate for curriculum development and reform. The third response has been at the level of 
implementation at local institution level broadly guided by national agreed curriculum frameworks and/or core competencies.

As potent and productive as these responses have been for their time, they have been enacted in something of a vacuum, that is, without an organizing theoretical framework for Indigenous health curricula. They exist outside of the logically sequenced teaching of more established subjects.

\section{'Intersectionality': A theoretical framework for health science education}

We suggest bringing a theoretical framework from public health and the social sciences to health professional education. The framework we advocate recognizes people's identities as plural and multidimensional [24] as well as 'intersecting' [25]. Such a framework is found in 'Intersectionality', a view of individuals as holding multiple social statuses (or, identities) that interact to shape individuals' health views/beliefs, needs and experiences [26] such as gender, ethnicity, sexuality, age and class. Through an intersectional lens, these multiple statuses are seen to intersect at the micro level of individual experience and in doing so reflect multiple interlocking systems (institutions) of privilege and oppression [27]. That is, dimensions of identities at an individual level (such as socioeconomic status, gender, ethnicity, sexuality) intersect with macro level social structures that result in multiple social inequalities manifested as racism, sexism, classism. It is important here to emphasize that an intersectional framework facilitates a view of both the advantages and disadvantages that different social locations afford each individual - and the power thus attributed to an individual.

The application of intersectionality in the social science and public health literature offers a framework for both research and education in the health professions, providing a way to engage with criticisms of and challenges to simplistic representations of race, gender and age. It goes beyond curriculum reform, too: educators and researchers are challenged to tackle institutional reform. Thus, institutions need to align themselves with and support improvements to the experiences of individuals and communities in need. '[A] 
central tenet of intersectionality is the insistence on applying theory to practice' [25], and an 'effort to improve society'. [28]

Intersectionality, with its antecedents in critical theory and critical race theory [1] as a means of bridging feminist and anti-racist discourses, argues for a multidimensional rather than a single-axis framework in which to consider experiences of discrimination. Discrimination has often encouraged interpretation of disadvantage through a single-axis (gender, race, class), but this view silences and marginalizes the most disadvantaged. In contrast, as Crenshaw argues, positioning the most marginalized at the center of the dialogue offers the dual advantage of addressing disadvantage across the range of experiences as well as dissuading compartmentalization of identity [1]. Thus, difference and diversity are examined across the intersection of axes.

Another important feature of the intersectional framework is the scope it offers for the 'interrogation of one's own blind spots and transforming them into analytic resources for further critical analysis', for 'asking the other question', that is, the question beyond the single-axis [24]. For example, an analysis of female medical students' experiences of gendered practices in a medical school [29] could, through an intersectional lens, have been expanded to include consideration of experiences related to professionalism, race, family choices and so on [30]. This approach has only recently been considered relevant to medical curriculum $[12,30,31]$.

\section{An 'Intersectional' approach to health professional education}

In emphasizing that intersectionality is action-oriented, Dill and Zambrana draw attention to four 'theoretical interventions' that could contribute to improved understandings and explanations of the lives of marginalized people [28]. We consider here how these four interventions - devised in the USA context resonate with emerging agendas elsewhere and illustrate each intervention with reference to Indigenous health in Australia.

Centering the experiences of people of color 
Intersectionality promotes the generation of knowledge and counter-narratives based on the experiences of previously excluded and oppressed groups experiences that diverge from the accepted and normalized narrative constructed from the experiences of the social elite. In this way, an intersectional framework makes visible both privilege and disadvantage.

In Australia, despite the poor health status of Indigenous people being a widely promoted national health priority, the voices and experiences of Indigenous Australians have been fundamentally marginalized in medical and other health professional education. Furthermore, historically, Australian Indigenous people have not participated in the medical profession as doctors [32]: the first Indigenous medical graduation in 1983 [33] took place over a century after medical graduations commenced in Australia. Indigenous people have been significantly marginalized from the health workforce and from the health-related knowledge economy, and comprise 0.2 percent of the health workforce [34] (whilst population parity is a somewhat crude target, it remains, at least, a measurable and challenging push target in terms of representation in the workforce). Indigenous medical student admissions have recently achieved population parity; however, graduation at this rate remains some years off [35].

We argue that there is a clear imperative to move those formerly marginalized voices and experiences of, in this case, Australian Indigenous peoples from the periphery to the center of the dialogue on health professional curriculum. An intersectional lens renders the previously invisible, visible, and recognizes the relationship between marginalized people and others: 'non-Indigenous' cannot remain the norm. Furthermore, the assumed neutrality that is usually associated with the biomedical sciences [35] can be questioned.

\section{Complicating identity}

The second component of an intersectional framework highlights the need to make identity more, rather than less, complicated. 
Both individual and group identity are complex - influenced and shaped not simply by a person's race, class, ethnicity, gender, physical ability, sexuality, religion, or nationality but by a combination of all of those characteristics [28].

We have noted earlier that courses focusing on one factor (age, gender, race) have often served to obscure difference and promote stereotyping and generalizations. Representations of identity in Problem Based Learning cases in medical education, for example, have prompted students to draw simplified onedimensional associations, so that the identification of the patient's race flagged a particular disease:

[Race] comes up ... with risk factors ... an African American woman who has sickle cell anemia, and just that association, because it's just one of the risk factors

—Fourth-year medical student [36]

A persistent tendency toward stereotyping across medical curricula occurs when associations of a specific ethnicity, gender or socioeconomic status are linked to certain disease states [8,37-39]. In our setting, Indigenous students in the health sciences have expressed concern about what they see in the curriculum as constructions of Indigenous identity related only to burden of disease, poverty and unproductive engagement with the health system. As mostly young, healthy and on the way to being well educated (as well as potentially affluent and influential) they find these representations alienating.

The structuring of health science learning around a bioscience or body systems approach is, of course based on sound logic. One result, however, is that the social complexities of race ... are rendered invisible' [36]. The alternative we recommend is a partnership approach to the application of an intersectional framework which would bring these complexities to the fore. Fully applied, such an approach would require an understanding of the issues of power that may have contributed to the apparent (though simplified) biomedical risk factors. 
There is a lack of evidence that current approaches to diversity are successful in affecting student's perceptions of diversity, their interactions with patients or, ultimately, cultural and ethnic disparities in health care $[12,14,20,21,31]$. We need to question the oversimplification inherent in models that perpetuate cultural stereotypes and diminish the importance of other aspects of status or identity that impact on health experiences $[12,40]$. One line of questioning concerns labels that homogenize identity and the complexities inherent within the group to which the label is applied [28]. In our example, the use of the term 'Indigenous' promotes simplification of a culturally, socio-economically and linguistically diverse group and hides diversity within a simplified social construct.

Unveiling power in interconnected structures of inequality

An intersectional framework insists on attention to power and how power operates to shape privilege and oppression. [25]

The impact of power on inequalities is recognized as both a force of oppression used by certain groups in society and an intangible entity that operates in society through four interrelated domains of power - structural, disciplinary, hegemonic, interpersonal [28]:

- Institutional structures perpetuate oppression through mechanisms such as policy. For Indigenous people, this operates via access to health and social services, opportunities for health education, and opportunity for, or exclusion from, social and economic justice.

- Certain disciplinary practices (ways of doing and being) sustain hierarchies and institutional structures. In medical education, the 'hidden curriculum' is seen to be more "concerned with replicating the culture of medicine than with the teaching of knowledge and techniques" [41]. The integration of Indigenous knowledge and medicines and the use of ngangkari (Aboriginal healers) are the exception rather than the norm in health professional curricula. 
- Hegemonic power operates via representations and cultural ideologies that shape identities and inform the structural and disciplinary domains. This influences the expectations of both Indigenous and non-Indigenous Australians and is seen to provide a justification for differential treatment in terms of policy and health care provision.

- Finally, power interactions in daily activity can be so subtle and normalized that they go unrecognized. A study of Indigenous medical students, for instance, showed an expectation that female students would become Aboriginal health workers or nurses [32].

Seen through this lens, power operates via multiple domains simultaneously, as 'Matrices of domination' [42]. In our example, to understand the antecedents of Indigenous health and the Indigenous patient's lived experience, a clinician needs to appreciate more than simple, one-dimensional representations and engage with the multi-layered, multifactorial elements, perhaps simultaneously including considerations of race, gender and the impacts of colonization.

Promoting Social Justice and Social Change.

Health professional education and the provision of health care are directly connected with social justice and social change.

[T]ransformation of knowledge and of individual lives is a fundamental aspect of intersectional work. ...Among ...[intersectional] scholars, discussions of social change focus not just on changing the society at large but also on changing structures of knowledge within institutions of higher learning and the relationship of colleges and universities to the society [28].

In this regard, the link between the influences of the curriculum on the learner and the subsequent effects on patient outcomes has not yet been demonstrated $[20,43,44]$. We suggest that social justice and social change need to be thought of as immediate curricula outcomes. For example, a student-led elective intended to 
address a hepatitis B health disparity for a high-risk population in San Francisco showed a demonstrable increase in health care access [45]. In another instance, increased medical practitioner numbers in areas of chronic practitioner shortages in rural and underserved areas has been encouraged through initiatives in community based medical education in Australia, the UK and Canada [46-48]. Looking to the situation of Indigenous Australians, a stronger position in health education and health care provision begins with recognition of their voices and experiences in order to diminish marginalization. What could follow is transformative scholarship that has the potential to shape knowledge within both higher education institutions and in the broader society served by those institutions [28].

\section{Practical Application of the Framework}

There are two important elements in the application of an intersectional framework: first, a partnership of specialist expertise is required to develop curriculum; second, a sequenced program of subject knowledge and skills is needed to work from the simple to the complex.

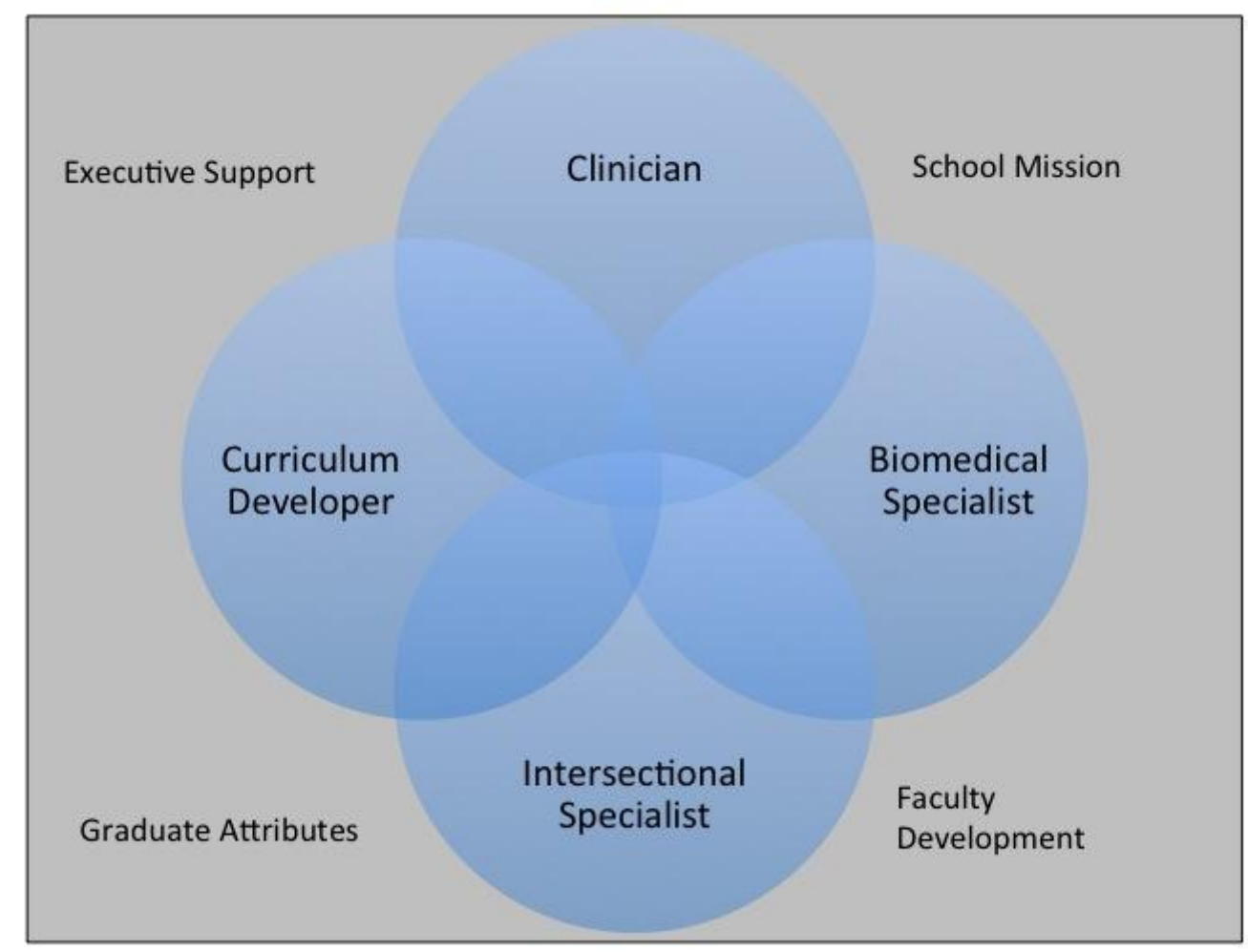


Figure 1: A partnership of experts for curriculum development

Figure 1 shows how our proposal for applying an intersectional framework requires a new partnership between the existing bioscience experts, clinical practice experts and specialists for the development of an intersectional curriculum. This partnership would operate in the context of and aligned with the school's mission statement, with support from the executive and through faculty development.

\begin{tabular}{|c|c|c|c|c|c|}
\hline & Phase 1 & Phase 2 & $\begin{array}{c}\text { Phase } \\
3\end{array}$ & Phase 4 & \\
\hline Intersectionality & \multicolumn{4}{|c|}{$\begin{array}{l}\text { Single axis issues extending to multiple axis issues } \\
\text { across the spectrum of the curriculum }\end{array}$} & $\begin{array}{l}\text { Graduate } \\
\text { Outcomes }\end{array}$ \\
\hline \multicolumn{6}{|c|}{ Simplicity } \\
\hline \multirow{2}{*}{ Pharmacology } & Physiology & $\begin{array}{c}\text { Body } \\
\text { Systems }\end{array}$ & \multirow{2}{*}{$\rightarrow \rightarrow$} & \multirow{2}{*}{$\begin{array}{c}\text { Clinical } \\
\text { Pharmacology }\end{array}$} & \multirow{2}{*}{$\begin{array}{l}\text { Graduate } \\
\text { Outcomes }\end{array}$} \\
\hline & $\begin{array}{c}\text { Bio- } \\
\text { Chemistry }\end{array}$ & Anatomy & & & \\
\hline
\end{tabular}

Figure 2: The sequencing of knowledge and skills

Figure 2 illustrates with two examples how the necessary simplicity and complexity of subject areas is dealt with in a course. In the same way that Pharmacology begins with the teaching of Physiology and Biochemistry and only later in the course introduces the complexities of Clinical Pharmacology, so Intersectionality can be structured. That is, in the early years, students would be introduced to single axis issues such as gender, ageing and ethnicity. In the later years in the course, such single axis issues are seen as intersecting via the teaching of a multiple axis perspective. In our example, and consistent with the genesis of Intersectionality in critical race theory, the multiple axis could be an Aboriginal woman. 


\section{Implications of adopting an intersectional framework}

In this paper we have explained the limitations of current health professional curriculum as it is constructed in and constrained by an organizing theoretical framework based solely on biosciences. We propose as part of the solution to persistent health disparity, the adoption of Intersectionality, an approach well established in related fields of higher education and scholarship. We have outlined the main constructs of that framework and now consider the five main implications of this transformative curriculum move.

1. A negotiated curriculum informed by an intersectional framework requires a nuanced, skilled, whole-of-curriculum and whole-of-institution approach. This would model the success of the Flexnerian approach, but would be applied within the complex and challenging space of diversity and disparities in health outcomes.

2. Both the 'what' and the 'how' of student evaluation would be different: students would be assessed on their nuanced understanding of diversity in people and populations whom they will later serve, as well as their successful incorporation of notions of social justice and advocacy into their academic and clinical work. The processes of evaluation would need to be aligned with all expected learning outcomes and teaching activities [49].

3. There is a particular role for faculty development in a School delivering a curriculum that is substantively underpinned by an intersectional framework. First, faculty development would attend to the process of aligning the formal, informal and hidden curriculum to promote a commitment to, for instance, issues related to the health of Indigenous peoples [50]. Second, faculty development would involve teachers in changing knowledge structures within and around institutions of higher education.

4. This transformative curriculum move requires executive-level acknowledgement of the inequitable outcomes of the power structures that have 
influenced the development of not just the goals and values of the curriculum but also the Faculty itself and the institution's relationships with local communities [51].

\section{Conclusions}

Health care systems and health care encounters are becoming increasingly globalized, complex and nuanced. This paper illustrates how an intersectional curriculum framework could advance health professional education to better serve diverse and marginalized people and populations. The example of Indigenous Australian peoples in this paper was employed to illustrate the need for and benefits of a new approach to what is a recognized national health priority. The new curriculum dialogue needs to acknowledge both the multiple statuses and identities each person brings to their interactions with the health care system and the various ways these identities connect individuals and groups to society's power structures. An intersectional framework offers an antidote to the focus on the adhoc, often simplistic - and unhelpful or even counterproductive - exotic Other. It requires a partnership of specialists to develop curriculum, a partnership to strengthen the power of biomedical teaching and learning to effect substantial change in diverse and marginalized populations. 


\section{References}

1. Crenshaw, K. (1989). Demarginalizing the intersection of race and sex: A black feminist critique of antidiscrimination doctrine, feminist theory, and antiracist politics. University of Chicago Legal Forum,14, 538-554.

2. Gustafson, D. L., \& Reitmanova, S. (2010). How are we 'doing' cultural diversity? A look across English Canadian undergraduate medical school programmes. Medical Teacher, 32(10), 816-823.

3. Seeleman, C., Suurmond, J., \& Stronks, K. (2009). Cultural competence: a conceptual framework for teaching and learning. Medical Education, 43(3), 229-237.

4. Besdine, R. W., Shield, R. R., McNicoll, L., Campbell, S. E., \& Wetle, T. (2011). Integrating and evaluating geriatrics in medical school: a novel approach for the challenge. Gerontology \& Geriatrics Education, 32(4), 295-308.

5. Shield, R. R., Farrell, T. W., Nanda, A., Campbell, S. E., \& Wetle, T. (2012). Integrating geriatrics into medical school: student journaling as an innovative strategy for evaluating curriculum. Gerontologist, 52(1), 98-110.

6. McGregor, A. J., \& Choo, E. (2012). Gender-specific Medicine: Yesterday's Neglect, Tomorrow's Opportunities. Academic Emergency Medicine,19(7), 861-865.

7. Risberg, G., Johansson, E. E., \& Hamberg, K. (2009). A theoretical model for analysing gender bias in medicine. International Journal for Equity in Health, 8(28), doi:10.1186/1475-9276-8-28

8. Verdonk, P., Benschop, Y., de Haes, H., Mans, L., \& Lagro-Janssen, T. (2009). 'Should you turn this into a complete gender matter?' Gender mainstreaming in medical education. Gender and Education, 21(6), 703-719.

9. Yut-Lin, W. (2009). Gender Competencies in the Medical Curriculum: Addressing Gender Bias in Medicine. Asia-Pacific Journal of Public Health, 21(4), 359-376.

10. Derenne, J., \& Roberts, L. (2010). Psychiatry's Role in Teaching Medical Students, Psychiatric Residents, and Colleague Physicians About Human Sexuality. Academic Psychiatry, 34(5), 321324.

11. Obedin-Maliver, J., Goldsmith, E. S., Stewart, L., White, W., Tran, E., Brenman, S., Lunn, M. R. (2011). Lesbian, Gay, Bisexual, and Transgender-Related Content in Undergraduate Medical Education. Journal of American Medical Association, 306(9), 971-977. 
12. Powell Sears, K. (2012). Improving cultural competence education: the utility of an intersectional framework. Medical Education, 46(6), 545-551.

13. Betancourt, J. R., \& Cervantes, M. C. (2009). Cross-cultural medical education in the united states: Key principles and experiences. Kaohsiung Journal of Medical Sciences, 25(9), 471-478. 14. Crosson, J. C., Deng, W., Brazeau, C., Boyd, L., \& Soto-Greene, M. (2004). Evaluating the effect of cultural competency training on medical student attitudes. Family medicine, 36(3), 199-203.

15. Lypson, M. L., Ross, P. T., \& Kumagai, A. K. (2008). Medical students' perspectives on a multicultural curriculum. Journal of the National Medical Association, 100(9), 1078-1083.

16. Perron, N. J., Perneger, T., Kolly, V., Dao, M. D., Sommer, J., \& Hudelson, P. (2009). Use of a computer-based simulated consultation tool to assess whether doctors explore sociocultural factors during patient evaluation. Journal of Evaluation in Clinical Practice, 15(6), 1190-1195.

17. Chheda, S., Hemmer, P. A., \& Durning, S. (2009). Teaching About Racial/Ethnic Health Disparities: A National Survey of Clerkship Directors in Internal Medicine. Teaching and Learning in Medicine, 21(2), 127-130.

18. Karnik, N. S., \& Dogra, N. (2010).The Cultural Sensibility Model: A Process-Oriented Approach for Children and Adolescents. Child and Adolescent Psychiatric Clinics of North America, 19(4), 719

19. Wear, D., Zarconi, J., Kumagai, A., Cole-Kelly, K. (2014). Slow Medical Education. Academic Medicine. First published online, doi: 10.1097/ACM.0000000000000581

20. Beagan, B. L. (2003). Teaching social and cultural awareness to medical students: "It's all very nice to talk about it in theory, but ultimately it makes no difference". Academic Medicine, 78(6), 605-614.

21. Whitehead, C., Kuper, A., \& Webster, F. (2012). The conceit of curriculum. Medical Education, 46(6), 534-536.

22. Flexner A. (1910). Medical Education in the United States and Canada. (The Carnegie Foundation for the Advancement of Teaching, Washington, DC).

23, Cooke, M., Irby, D.M., O'Brien, B.C. (2010) Educating Physicians: A call for reform of medical school and residency. (Jossey Bass, Stanford). 
24. Davis, K. (2008). Intersectionality as buzzword: A sociology of science perspective on what makes a feminist theory successful. Feminist Theory, 9(1), 67-85.

25. Jones, S. R., \& Wijeyesinghe, C. L. (2011). The promises and challenges of teaching from an intersectional perspective: Core components and applied strategies. New Directions for Teaching and Learning, 2011(125), 11-20.

26. Sears KP. (2012). Improving cultural competence education: the utility of an intersectional framework. Medical Education.46(6):545-51.

27. Bowleg L. (2012). The problem with the phrase women and minorities: Intersectionality - an important theoretical framework for public health. American Journal of Public Health. 102(7):1267-73.

28. Dill, B., \& Zambrana, R. (2009). Critical thinking about inequality: an emerging lens. In B. Dill \& R. Zambrana (Eds.), Emerging Intersections: Race, Class, and Gender in Theory, Policy, and Practice (pp. 1-21). (New Brunswick, Rutgers University Press)

29. Babaria, P., Bernheim S., Nunez-Smith M. (2011). Gender and the pre-clinical experiences of female medical students: A taxonomy. Medical Education, 45(3), 249-60

30. Tsouroufli, M., Rees, C. E., Monrouxe, L. V., \& Sundaram, V. (2011). Gender, identities and intersectionality in medical education research. Medical Education, 45(3), 213-216.

31. McLean, M. (2012). Broadening our perceptions of diversity in medical education: using multifocal lenses. Medical Education, 46(6), 536-538.

32. Garvey, G., Rolfe, I. E., Pearson, S.-A., \& Treloar, C. (2009). Indigenous Australian medical students' perceptions of their medical school training. Medical Education, 43(11), 1047-1055.

33. Anderson, I. The knowledge economy and Aboriginal health development. The University of Melbourne, Faculty of Medicine, Dentistry and Health Sciences Dean's lecture series, 2008.

34. Australian Indigenous Doctors Association. 2012. Aboriginal and Torres Strait Islander medical student numbers jump. Medical Deans Australia and New Zealand, Media Release, Aug 2012 [accessed online 19 January, 2015 http://www.medicaldeans.org.au/aboriginal-andtorres-strait-islander-medical-student-numbers-jump.html

35. Taylor, J.S. (2003). Confronting "culture" in medicine's "culture of no culture". Academic Medicine, 78(6):555-9. 
36. MacLeod, A. (2011). Six Ways Problem-Based Learning Cases Can Sabotage Patient-Centered Medical Education. Academic Medicine, 86(7), 818-825.

37. Kai, J., Bridgewater, R., \& Spencer, J. (2001). ' "Just think of TB and Asians", that's all I ever hear': Medical learners' views about training to work in an ethnically diverse society. Medical Education, 35(3), 250-256.

38. Loudon, R. F., Anderson, P. M., Gill, P. S., \& Greenfield, S. M. (1999). Educating medical students for work in culturally diverse societies. Journal of American Medical Association, 282(9), 875-880.

39. McLean, D., Atyeo, J., Sarkar, S., Robinson, J., Davison, A., \& Barrie, S. (2000). Cross-cultural teaching in medical radiation technology : degree conversion course in Singapore. Focus on Health Professional Education, 2(1), 67-74.

40. Boutin-Foster, C., Foster, J. C., \& Konopasek, L. (2008). Viewpoint: Physician, Know Thyself: The Professional Culture of Medicine as a Framework for Teaching Cultural Competence. Academic Medicine, 83(1), 106-111.

41. Hafferty FW, Franks R. (1994). The hidden curriculum, ethics teaching and the structure of medical education. Academic Medicine, 69(11), 861-871

42. Collins, P. (2000). Black feminist thought : knowledge, consciousness, and the politics of empowerment (2nd ed). (New York: Routledge)

43. Larson, B., Herx, L., Williamson, T., \& Crowshoe, L. (2011). Beyond the barriers: family medicine residents' attitudes towards providing Aboriginal health care. Medical Education, 45(4), 400-406.

44. Ewen SC, Paul DJ, Bloom GL. (2012). Do indigenous health curricula in health science education reduce disparities in health care outcomes? Medical Journal of Australia, 197(1):50-2.

45. Sheu, L.C., Toy, B.C., Kwahk, E., Yu, A., Adler, J., Lai, C.J. (2010). A Model for Interprofessional Health Disparities Education: Student-Led Curriculum on Chronic Hepatitis B Infection. Journal of General Internal Medicine, 25 (Suppl 2) 140-145.

46. Strasser, R. P. (2010). Community engagement: a key to successful rural clinical education. Rural \& Remote Health, 10(3), 1543. 
47. Worley, P., Silagy, C., Prideaux, D., Newble, D., \& Jones, A. (2000). The Parallel Rural

Community Curriculum: an integrated clinical curriculum based in rural general practice. Medical Education, 34(7), 558-565.

48. Rabinowitz, H. K. (1993). Recruitment, retention, and follow-up of graduates of a program to increase the number of family physicians in rural and underserved areas. New England Journal of Medicine, 328(13), 934-939.

49. Biggs, J. (2003). Teaching for Quality at University. (The Society for Research into Higher Education and Open University Press).

50. Ewen, S., Mazel, O., Knoche, D. (2012). Exposing the hidden curriculum influencing medical education on the health of indigenous people in Australia and New Zealand: The role of the Critical Reflection Tool. Academic Medicine, 87(2), 200-205.

51. Apple, M.W. (1971). The hidden curriculum and the nature of conflict. Interchange, 2(4): 27 40. 


\section{University Library}

\section{- M M I N E R VA A gateway to Melbourne's research publications}

Minerva Access is the Institutional Repository of The University of Melbourne

Author/s:

Ewen, S;Barrett, J;Howell-Meurs, S

Title:

Health Disparity and Health Professional Education: A New Approach

Date:

2016-06-01

Citation:

Ewen, S., Barrett, J. \& Howell-Meurs, S. (2016). Health Disparity and Health Professional Education: A New Approach. Medical Science Educator, 26 (2), pp.247-253. https:// doi.org/10.1007/s40670-016-0230-2.

Persistent Link:

http://hdl.handle.net/11343/283009 